University of Wollongong

Research Online

Faculty of Engineering and Information

Faculty of Engineering and Information

Sciences - Papers: Part A

Sciences

2001

Numerical investigation of the angle of repose of monosized spheres

Y C. Zhou

University of New South Wales

B H. Xu

University of New South Wales

Aibing Yu

University of New South Wales

Paul Zulli

University of Wollongong, paulz@uow.edu.au

Follow this and additional works at: https://ro.uow.edu.au/eispapers

Part of the Engineering Commons, and the Science and Technology Studies Commons

Research Online is the open access institutional repository for the University of Wollongong. For further information contact the UOW Library: research-pubs@uow.edu.au 


\title{
Numerical investigation of the angle of repose of monosized spheres
}

\begin{abstract}
This paper presents a numerical study of the angle of repose, a most important macroscopic parameter in characterizing granular materials, by means of a modified distinct element method. Emphasis is given to the effect of variables related to factors such as particle characteristics, material properties, and geometrical constraints. The results show that sliding and rolling frictions are the primary reasons for the formation of a sandpile; particle size and container thickness significantly influence the angle of repose; and the angle of repose is not so sensitive to density, Poisson's ratio, damping coefficient, and Young's modulus. Increasing rolling friction coefficient or sliding friction coefficient increases the angle of repose. Conversely, increasing particle size or container thickness decreases the angle of repose. The underlying mechanisms for these effects are discussed in terms of particle-particle and particle-wall interactions.
\end{abstract}

\section{Keywords}

monosized, spheres, investigation, repose, numerical, angle

Disciplines

Engineering | Science and Technology Studies

\section{Publication Details}

Zhou, Y. C., Xu, B. H., Yu, A. B. \& Zulli, P. (2001). Numerical investigation of the angle of repose of monosized spheres. Physical Review E: Statistical, Nonlinear, and Soft Matter Physics, 64 021301-1-021301-8. 


\title{
Numerical investigation of the angle of repose of monosized spheres
}

\author{
Y. C. Zhou, B. H. Xu, and A. B. Yu* \\ Centre for Computer Simulation and Modelling of Particulate Systems, School of Materials Science and Engineering, \\ The University of New South Wales, Sydney, NSW 2052, Australia \\ P. Zulli \\ BHP Steel Research Laboratories, P.O. Box 202, Port Kembla, NSW 2505, Australia
}

(Received 29 September 2000; revised manuscript received 5 April 2001; published 18 July 2001)

\begin{abstract}
This paper presents a numerical study of the angle of repose, a most important macroscopic parameter in characterizing granular materials, by means of a modified distinct element method. Emphasis is given to the effect of variables related to factors such as particle characteristics, material properties, and geometrical constraints. The results show that sliding and rolling frictions are the primary reasons for the formation of a sandpile; particle size and container thickness significantly influence the angle of repose; and the angle of repose is not so sensitive to density, Poisson's ratio, damping coefficient, and Young's modulus. Increasing rolling friction coefficient or sliding friction coefficient increases the angle of repose. Conversely, increasing particle size or container thickness decreases the angle of repose. The underlying mechanisms for these effects are discussed in terms of particle-particle and particle-wall interactions.
\end{abstract}

DOI: 10.1103/PhysRevE.64.021301

PACS number(s): 45.70.Cc, 45.50.-j, 45.70.Mg

\section{INTRODUCTION}

The angle of repose is one of the most important macroscopic parameters in characterizing the behavior of granular materials. It is related to many important phenomena, including avalanching [1-3], stratification [4,5], and segregation [6-8], and is therefore a research focus for years. It has been found that the angle of repose strongly depends on material properties such as sliding friction coefficient $[9,10]$, rolling friction coefficient [11] and density of particles [12], and particle characteristics such as size $[8,13]$ and shape $[12,14]$, in addition to the way to form a heap or sandpile $[15,16]$.

The bulk behavior of a particle system depends on the collective interactions of individual particles, and hence particle scale analysis plays a critical role in elucidating the underlying mechanisms of the effects mentioned above. In the past, various modeling techniques have been used to investigate the behavior of particles in granular media at such a scale, including Monte Carlo (MC) [17,18], cellular automaton (CA) $[19,20]$, and distinct element method (DEM) $[21,22]$. Amongst these techniques, DEM is probably the most realistic one, because it explicitly takes into account not only the geometrical factors but also the forces involved in the formation of a sandpile. However, previous DEM studies are largely limited to two-dimensional and also suffer the problem of stabilizing a heap composed of spheres as there is no mechanism to stop spheres from rolling $[9,23]$. As a result, arbitrary treatments have to be implemented in a simulation, which may distort the reality and generate inaccurate information.

We have recently found that a stable three-dimensional heap can be formed naturally with the incorporation of a rolling friction model into the DEM [11]. The resulting

\footnotetext{
*Corresponding author. FAX: +61 29385 5956. Email address:
} a.yu@unsw.edu.au modified DEM would provide an effective way to study the angle of repose and the complex dynamic internal state of sandpiles under well controlled conditions. As a first step in this direction, this paper presents a detailed parametric study of the dependence of the angle of repose on key variables such as rolling friction coefficient, sliding friction coefficient, particle size, and container thickness.

\section{SIMULATION METHOD}

\section{A. Discrete particle simulation}

The simulations were performed based on the DEM originally proposed by Cundall and Strack [21] but modified by incorporating a rolling friction model in the rotational equation of a particle. Since this modified DEM has been detailed elsewhere [11], this section only outlines its key features for the purpose of completeness.

According to this model, the translational and rotational motions of particle $i$ in a system at time $t$, caused by its interactions with neighboring particles or walls, can be described by the following equations:

$$
m_{i} \frac{d \mathbf{V}_{i}}{d t}=m_{i} \mathbf{g}+\sum_{j=1}^{k_{i}}\left(\mathbf{F}_{c, i j}+\mathbf{F}_{d, i j}\right)
$$

and

$$
I_{i} \frac{d \boldsymbol{\omega}_{i}}{d t}=\sum_{j=1}^{k_{i}}\left(\mathbf{T}_{i j}+\mathbf{M}_{i j}\right)
$$

where $m_{i}, I_{i}, \mathbf{V}_{i}$, and $\boldsymbol{\omega}_{i}$ are, respectively, the mass, moment of inertia, translational, and rotational velocities of particle $i$. As shown in Fig. 1, the forces involved are: gravitational force $m_{i} \mathbf{g}$, and interparticle forces between particles $i$ and $j$, which include the contact force $\mathbf{F}_{c, i j}$, and viscous contact damping force $\mathbf{F}_{d, i j}$. These interparticle forces are 


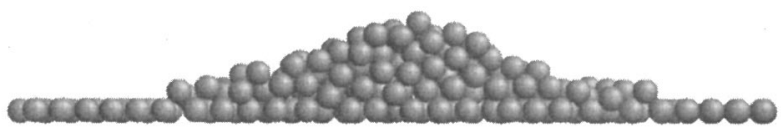

(a)

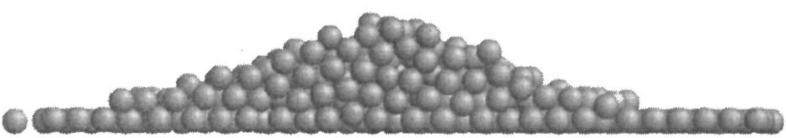

(b)

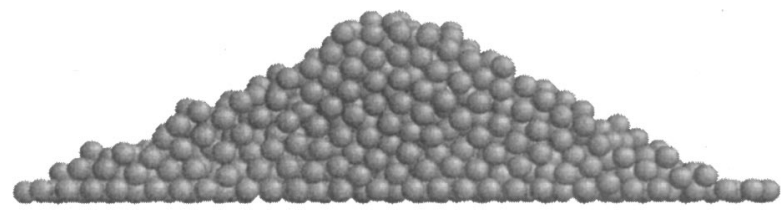

(c)

FIG. 3. Sandpiles simulated with size $d=10 \mathrm{~mm}$ and different friction coefficients: (a) $\mu_{s, \mathrm{pp}}=0.4, \mu_{r, \mathrm{pp}}=0.01 \mathrm{~mm}$; (b) $\mu_{s, \mathrm{pp}}$ $=0.2, \mu_{r, \mathrm{pp}}=0.05 \mathrm{~mm}$; (c) $\mu_{s, \mathrm{pp}}=0.4, \mu_{r, \mathrm{pp}}=0.05 \mathrm{~mm}$. The angle of repose $\theta$ of a sandpile is obtained by averaging the measurement at two sides with their tail ignored.

terial properties, and geometric conditions. Table II lists the variables considered in this study. For convenience, unless otherwise specified, the effect of a variable was considered within a certain range while other variables were fixed, giving a so-called base condition (Table II). Totally, 110 simulations were conducted in this work.

\section{RESULTS AND DISCUSSION}

Essentially, all the variables listed in Table II can affect the angle of repose except for the time step that is mainly used to control the numerical stability. However, trial simulations indicated that the angle of repose slightly increases as particle density or Poisson ratio increases, and has no obvious change with damping coefficient and Young's modulus for the ranges considered (Table II). The discussion below is

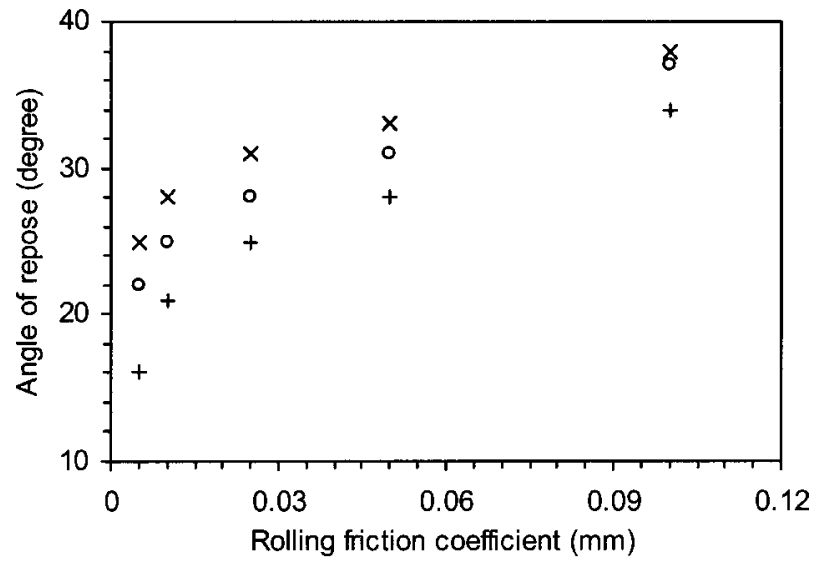

FIG. 4. Angle of repose as a function of rolling friction coefficient with different sliding friction coefficients:,$+ \mu_{s, \mathrm{pp}}=0.4 ; \bigcirc$, $\mu_{s, \mathrm{pp}}=0.5 ; \times, \mu_{s, \mathrm{pp}}=0.6$.

therefore only focused on the variables that have significant effects on the angle of repose.

\section{A. Effect of rolling friction coefficient}

Rotation and translation are the primary states of motion of a particle. The rolling and sliding frictions provide an effective mechanism to control the two motions and largely determine the individual contact stability in a sandpile. Therefore, they have significant effects on the angle of repose. To quantify the effect of rolling friction coefficient $\mu_{r, \mathrm{pp}}$ on the angle of repose, simulations were conducted for $10 \mathrm{~mm}$ spherical particles with different $\mu_{r, \mathrm{pp}}$ and different sliding friction coefficient $\mu_{s, \mathrm{pp}}$. As shown in Figs. 3(a) and 3(c), a large rolling friction coefficient gives a large angle of repose. Figure 4 shows the relationship between the angle of repose and rolling friction coefficient for different sliding friction coefficients. Obviously, increasing rolling friction coefficient can increase the angle of repose for a given slid-

TABLE II. Variables considered, their base values and ranges, and corresponding angle of repose.

\begin{tabular}{llllc}
\hline \hline Name of variable & \multicolumn{1}{c}{ Symbol } & \multicolumn{1}{c}{ Base value } & Variable range & Angle of repose $\left(^{\circ}\right)$ \\
\hline Number of particles & $N$ & $2000^{\mathrm{a}}$ & \\
Time step & $T$ & $10^{-6}-5 \times 10^{-5} \mathrm{sec}$ & & \\
Particle diameter & $d$ & $10 \mathrm{~mm}$ & $2-10 \mathrm{~mm}$ & {$[38,28]$} \\
Rolling friction & $\mu_{r, \mathrm{pp}}$ & $0.05 \mathrm{~mm}$ & $0-0.1 \mathrm{~mm}$ & {$[0,34]$} \\
coefficients & & & & \\
& $\mu_{r, \mathrm{pw}}$ & $2 \mu_{r, \mathrm{pp}}$ & $0-0.2 \mathrm{~mm}$ & {$[0,30]$} \\
Sliding friction & $\mu_{s, \mathrm{pp}}$ & 0.4 & $0-0.6$ & {$[0,33]$} \\
coefficient & & & & {$[0,28]$} \\
& $\mu_{s, \mathrm{pw}}$ & $1.5 \mu_{s, \mathrm{pp}}$ & $0-0.6$ & {$[28,19]$} \\
Container thickness & $\xi(=w / d)$ & $4 d$ & $4 d-24 d$ & {$[27,31]$} \\
Density & $\rho$ & $2500 \mathrm{~kg} / \mathrm{m}^{3}$ & $500-5000 \mathrm{~kg} / \mathrm{m}^{3}$ & $26,30]$ \\
Poisson ratio & $v$ & 0.3 & $0.1-0.7$ & $28 \pm 1$ \\
Young's modulus & $E$ & $2.16 \times 10^{6} \mathrm{~N} / \mathrm{m}^{2}$ & $10^{5}-10^{8} \mathrm{~N} / \mathrm{m}^{2}$ & $0.1-0.8$ \\
Damping coefficient & $c\left(c_{t}=c_{n}\right)$ & 0.4 & & \\
\hline \hline
\end{tabular}

${ }^{\mathrm{a}} N$ increases as $\xi$ increases. 


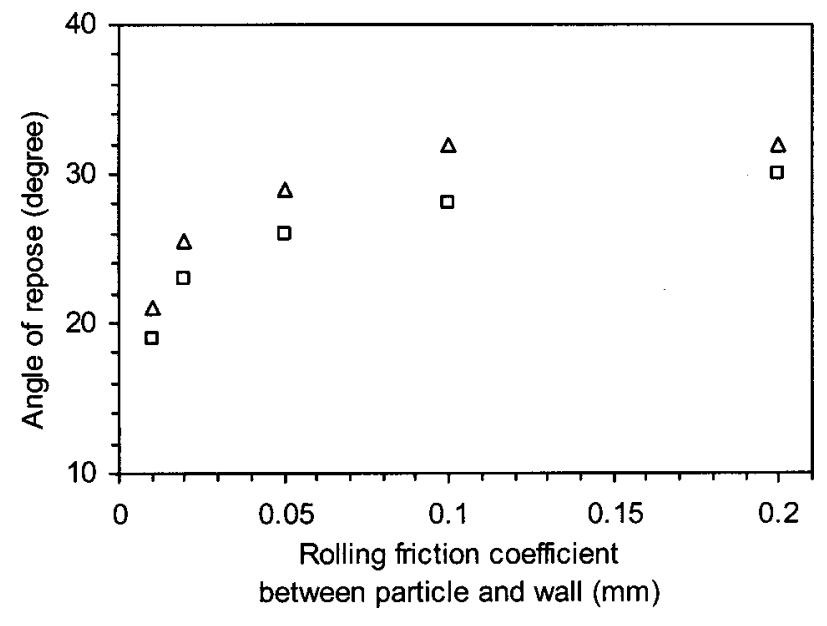

FIG. 5. Angle of repose as a function of rolling friction coefficient between particle and wall with $\mu_{s, \mathrm{pp}}=0.4$ and different $\mu_{r, \mathrm{pp}}$ : $\square, \mu_{r, \mathrm{pp}}=0.05 \mathrm{~mm} ; \triangle, \mu_{r, \mathrm{pp}}=0.1 \mathrm{~mm}$.

ing friction coefficient and particle size. Similar trends have also been observed for other sized particles.

The rolling friction between particle and wall is also an important parameter and gives a torque resistant to the rotational motion of particles on the middle plate and front and rear walls of the container. As shown in Fig. 5, increasing the rolling friction between particle and wall can significantly increase the angle of repose. Figures 4 and 5 suggest that the rolling friction between particles $\mu_{r, \mathrm{pp}}$ and the rolling friction between particle and wall $\mu_{r, \mathrm{pw}}$ are both important in controlling the angle of repose. This is because a large rolling friction coefficient means a large resistance force to the rotational motion of spheres, which provides an effective mechanism to consume the kinetic energy and stop the rotational motion of spheres, leading to the formation of a sandpile of high potential [11]. A rolling spherical particle will keep rolling if no rolling friction is involved. It also applies to a system of particles, thus no stable heap of spheres can be formed on a flat plate without rolling friction. This explains why extra conditions had to be used to stop particle movement in the previous dynamic simulation of sandpile formation $[23,24]$.

\section{B. Effect of sliding friction coefficient}

Sliding friction governs the translational motion of particles. A large sliding friction coefficient can tolerate a large magnitude of the elastic deformation in the tangential direction and enhance the stability of individual contacts among particles and between particle and wall. Therefore, the effect of sliding friction coefficient is similar to that of rolling friction coefficient. That is, a large sliding friction coefficient gives a large angle of repose. This is indeed the case as shown in Figs. 3(b), 3(c), and 6.

Notably, the results in Fig. 6 indicate that the angle of repose increases nonlinearly with increasing sliding friction coefficient. This is different from the linear relationship obtained by Lee and Herrmann [9]. There are a number of reasons for this. First, the range covered in the study of Lee and Herrmann is $\mu_{s} \leqslant 0.2$, smaller than the present range

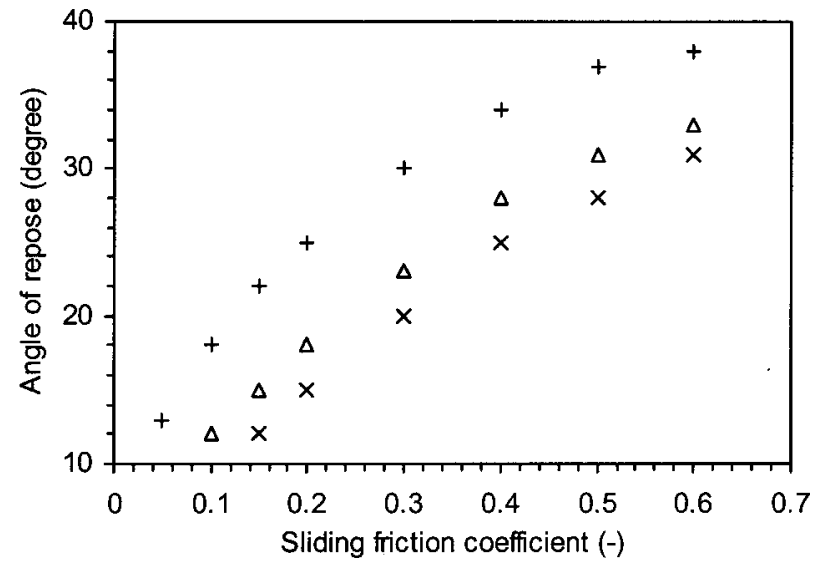

FIG. 6. Angle of repose as a function of sliding friction coefficient with different rolling friction coefficients: $\times, \mu_{r, \mathrm{pp}}$ $=0.025 \mathrm{~mm} ; \triangle, \mu_{r, \mathrm{pp}}=0.05 \mathrm{~mm} ;+, \mu_{r, \mathrm{pp}}=0.1 \mathrm{~mm}$.

$\mu_{s} \leqslant 0.6$. Second, the present work is three dimensional and involves the use of a rolling friction model, while the simulation of Lee and Herrmann is two dimensional and completely ignores the rotational motion of spheres. Third, the operational conditions for the two simulations are not the same. Lee and Herrmann formed a sandpile by releasing particles from one side of the box.

In a sufficiently large pile, sliding friction coefficient between particles determines the angle of repose as stated by Lee and Herrmann [9]. However, our numerical study indicates that the angle of repose was also influenced significantly by sliding friction coefficient between particle and wall $\mu_{s, p w}$, as shown in Fig. 7. No stable heap can be formed without sliding friction between particle and wall. This result is consistent with the previous experimental observation that the angle of repose is obviously higher on a high frictional surface than on a smooth surface [16]. In practice, a large sliding coefficient is often coupled with a large rolling friction coefficient, although the latter is also directly related to particle shape. The results in Figs. 4-7 suggest that both

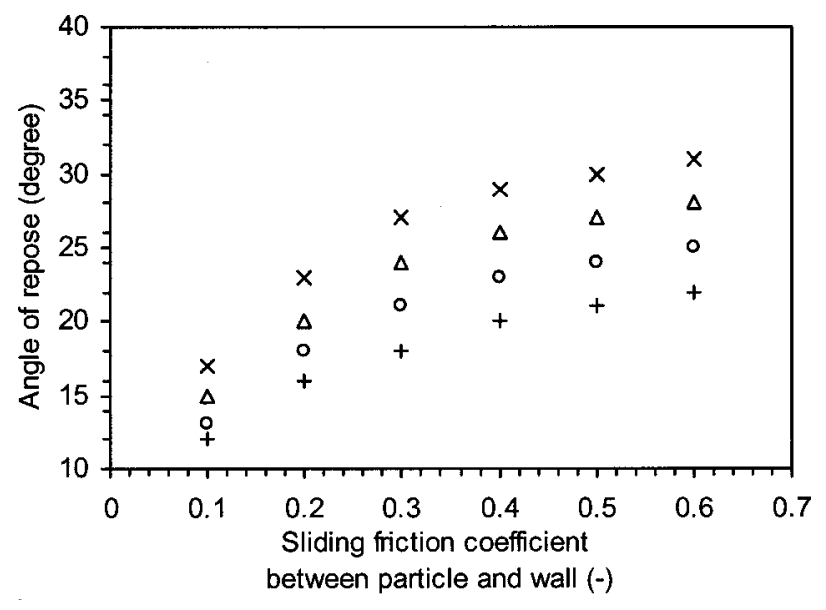

FIG. 7. Angle of repose as a function of sliding friction coefficient between particle and wall with $\mu_{r, \mathrm{pp}}=0.05 \mathrm{~mm}$ and different $\mu_{s, \mathrm{pp}}:+, \mu_{s, \mathrm{pp}}=0.2 ; \bigcirc, \mu_{s, \mathrm{pp}}=0.3 ; \triangle, \mu_{s, \mathrm{pp}}=0.4 ; \times, \mu_{s, \mathrm{pp}}$ $=0.5$. 


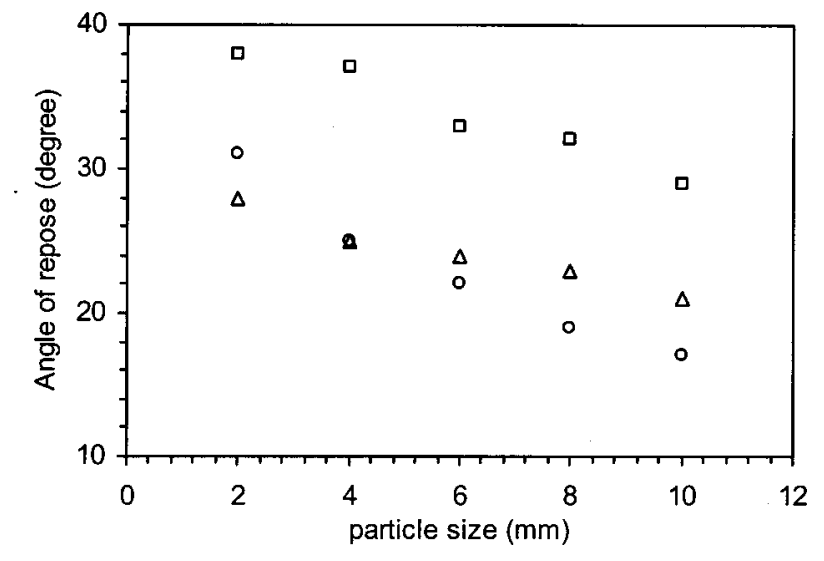

FIG. 8. Angle of repose vs particle size, with $w / d=4$ and different $\mu_{s, \mathrm{pp}}$ and $\mu_{r, \mathrm{pp}}: \bigcirc, \mu_{r, \mathrm{pp}}=0.05 \mathrm{~mm}$ and $\mu_{s, \mathrm{pp}}=0.2 ; \Delta$, $\mu_{r, \mathrm{pp}}=0.01 \mathrm{~mm}$ and $\mu_{s, \mathrm{pp}}=0.4 ; \square, \mu_{r, \mathrm{pp}}=0.05 \mathrm{~mm}$ and $\mu_{s, \mathrm{pp}}$ $=0.4$.

friction coefficients affect the stability of a sandpile and their proper combination is key to generating results comparable to those physically measured.

\section{Effect of particle size}

The effect of particle size on the angle of repose has been studied by a number of investigators with a general conclusion that increasing particle size will decrease the angle of repose [12-14]. This relationship has also been observed in the present study, as shown in Fig. 8. However, it appears that the significance of this size effect varies with simulation conditions, the sliding and rolling friction coefficients in particular.

Carstensen and Chan [13] suggested that the size effect is related to two factors: particle cohesive force and sliding friction coefficient. In particular, under the assumptions that the cohesive force is proportional to particle size and the coefficient of sliding friction decreases with particle size, these authors obtained an equation to relate the angle of repose to particle size. However, their approach does not apply to the present study that concerns with coarse, cohesionless spheres and uses constant sliding and rolling friction coefficients in quantifying the effect of particle size. Therefore, the underlying mechanism should be further explored.

The discussion in Secs. III A and III B clearly indicates that sliding and rolling frictions are primary factors in controlling the translational and rotational movement of a particle and hence the formation and stability of a sandpile. Therefore, the effect of particle size on the angle of repose can be depicted by examining its effect on the total sliding friction force $\mathbf{F}_{t, i}=\Sigma_{j=1}^{k_{i}}\left(\mathbf{F}_{c t, i j}+\mathbf{F}_{d t, i j}\right)$ and rolling friction torque $\mathbf{M}_{i}=\Sigma_{j=1}^{k_{i}}\left(\mu_{r}\left|\mathbf{F}_{c n, i j}\right|\right) \hat{\boldsymbol{\omega}}_{i}$ acting on a particle. Obviously, $\mathbf{F}_{t, i}$ and $\mathbf{M}_{i}$ vary temporally and spatially, as will be discussed in our future work. For simplicity, we only considered the particles above the middle plate and obtained their averaged force and torque in the present analysis. Figures 9 and 10 show the variation of the averaged magnitudes of $\mathbf{F}_{t}$ and $\mathbf{M}$ during the discharge process for a given particle size. Obviously, both magnitudes decrease with time and finally

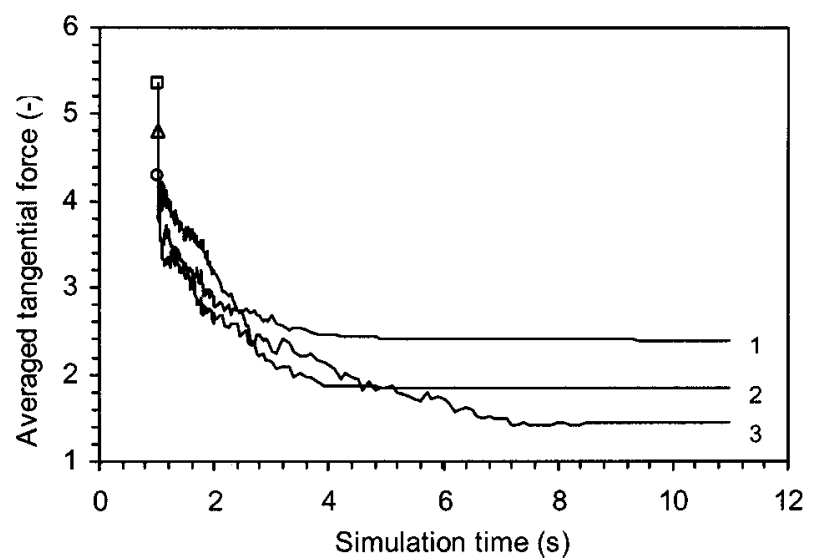

FIG. 9. Dimensionless average tangential force, defined as $\mathrm{F}_{t}$ $=1 / n\left|\sum_{i=1}^{n}\left(\sum_{j=1}^{k_{i}}\left(\mathbf{F}_{c t, i j}+\mathbf{F}_{d t, i j}\right)\right)\right| /(n m g)$ ( $n$ is number of particles above the middle plate), vs simulation time for different sized particles: line $1, d=5 \mathrm{~mm}$; line $2, d=10 \mathrm{~mm}$; line $3, d=20 \mathrm{~mm}$.

reach a steady value corresponding to a stable sandpile. Figures 11 and 12 show the plot of the final magnitude $\mathbf{F}_{t}$ and $\mathbf{M}$ as a function of particle size, after nondimensionalisation to be comparative. The results indicate that both the dimensionless sliding friction force and rolling friction torque decrease with the increase of particle size. However, the decreasing rate for the rolling friction torque is much more significant than that for the sliding friction force. Therefore, it is likely that for coarse spheres, particle size affects the angle of repose mainly through its effect on rolling friction, rather than sliding friction as suggested by Carstensen and Chan [13].

\section{Effect of container thickness}

So far, we have discussed the effects of rolling and sliding friction coefficients and particle size on the angle of repose. The container used is scaled by a particle diameter, and its thickness is four times of particle diameter as illustrated in Fig. 2. The front and rear walls set up an additional constraint to limit the mobility of particles in contact with the

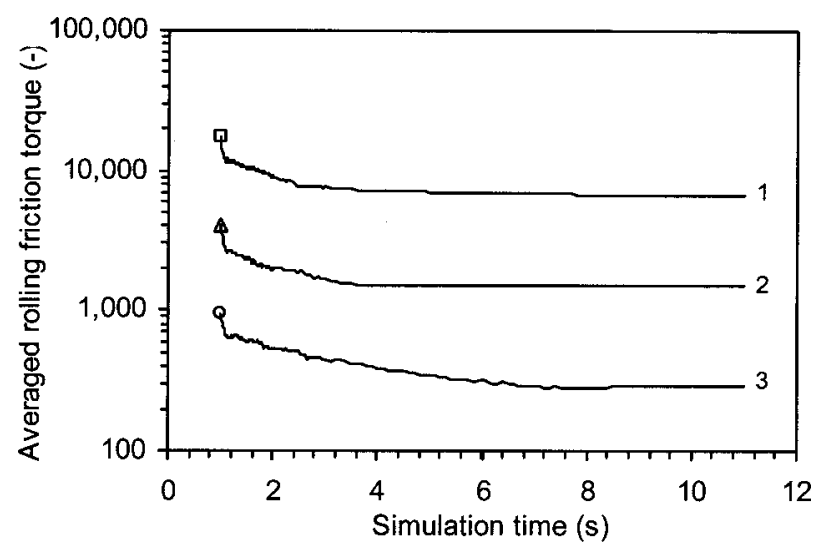

FIG. 10. Dimensionless average rolling friction torque, defined as $M=\sum_{i=1}^{n}\left(\sum_{j=1}^{k_{i}}\left(\mu_{r}\left|\mathbf{F}_{c n, i j}\right|\right)\right) /(n I)$, vs simulation time for different sized particle: line $1, d=5 \mathrm{~mm}$; line $2, d=10 \mathrm{~mm}$; line $3, d$ $=20 \mathrm{~mm}$. 


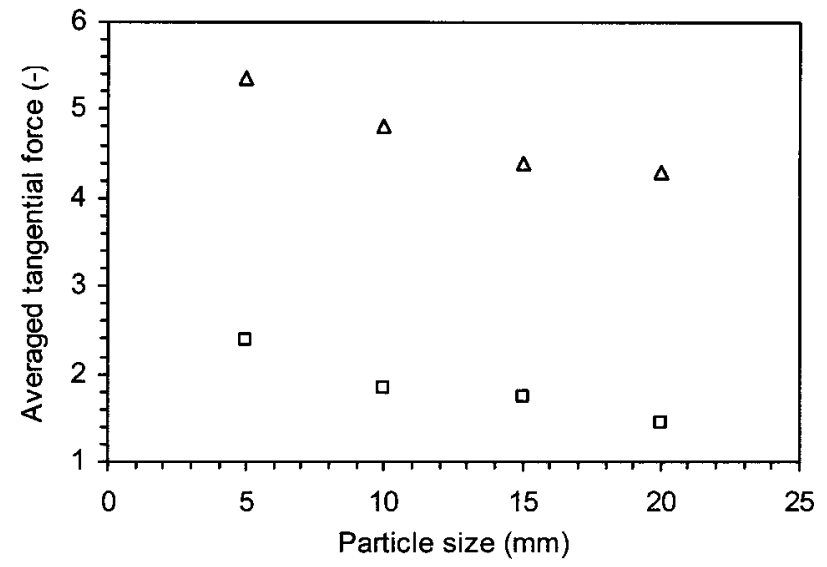

FIG. 11. Dimensionless average tangential force vs particle size at different simulation time; $\triangle, 1 \mathrm{sec}$ (just before opening the two outlets): $\square, 11 \mathrm{sec}$ (after forming a stable sandpile).

walls, which can propagate into the particle assembly to affect the angle of repose. In fact, Figs. 5 and 7 show changing rolling or sliding friction coefficient between particle and wall varies the angle of repose, implying that the front and rear walls, like the middle plate, have their effect here.

To quantify this effect, simulations were performed using different container thickness. Figure 13 shows that increasing the container thickness $w$ decreases the angle of repose for given simulation conditions. However, when the thickness is larger than a critical value, about 20 particle diameters, a constant angle of repose can be obtained, this corresponding to a situation without any front and rear wall effect. The trend is very much similar to that observed by Grasselli and Herrmann [15] who recently studied this effect for spheres ranging from 112 to $400 \mu \mathrm{m}$ in size. These authors, however, observed a much larger critical container thickness (equal to about 180 particle diameter). This difference, together with others to be discussed later, can be attributed to different experimental conditions in size range and interparticle forces. The cohesive force between particles, which can result from the van der Waals force for dry particles less than

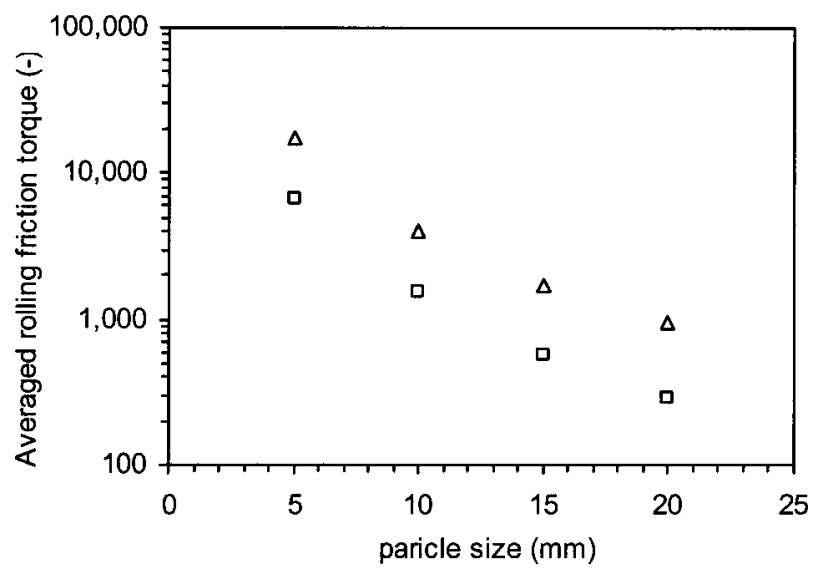

FIG. 12. Dimensionless average rolling friction torque vs particle size at different simulation time: $\triangle, 1 \mathrm{sec}$ (just before opening the two outlets): $\square, 11 \mathrm{sec}$ (after forming a stable sandpile).

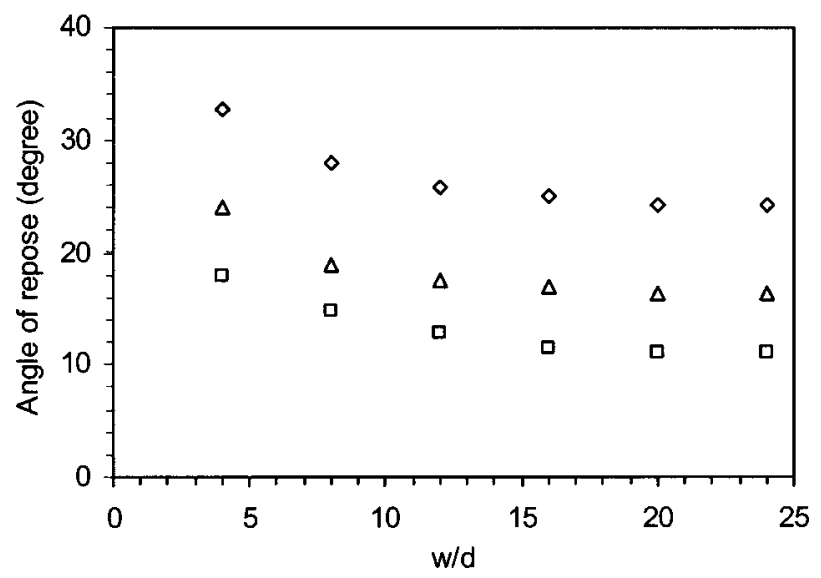

FIG. 13. The angle of repose against container thickness $(w / d)$ simulated when $\mu_{r, \mathrm{pp}}=0.025 \mathrm{~mm}$ and $\mu_{s, \mathrm{pp}}=0.4$ with different particle sizes: $\diamond, d=2 \mathrm{~mm} ; \triangle, d=5 \mathrm{~mm} ; \square, d=10 \mathrm{~mm}$.

$100 \mu \mathrm{m}$ or the capillary force for humidified particles, affect the piling behavior of the particles significantly $[13,16,25-$ 27].

Theoretically speaking, any particle in a stable sandpile must be at an equilibrium state and the sandpile as a whole, supported by its geometrical boundaries, must also be at equilibrium. The formation of strong force arches formed in boundary regions, therefore, must be a major factor responsible for the observed thickness effect. It was argued that the smaller the thickness, the easier to form arches and hence the larger the angle of repose [15]. To validate this consideration, three-dimensional force networks were constructed based on the present simulated results. The analysis is focused on the normal contact force because, as implied by the equations listed in Table I, other forces are related to this force. Because of the dominant role played by the gravity, particles are mainly supported by a complicated force network propagating into a sandpile from the middle plate, as shown in Fig. 14 where the centres of two contact spheres are linked by sticks of different thickness that is proportional to the magnitude of a normal force. This is always the case irrespective to the thickness of the container. However, as shown in Fig. 15 , if the thickness is small, large forces can also be observed close to the front and rear walls. These force arches, like those originated from the bottom wall, will also contribute to the stability of a sandpile. However, such force arches vanish rapidly with increasing container thickness. In fact, for a pile of thickness 16 particle diameter, the spatial distribution of the normal forces becomes rather uniform along

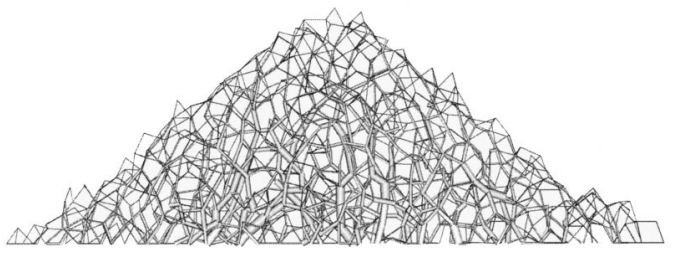

FIG. 14. Front view of the normal force network in a sandpile simulated with $w=4 d, \mu_{r, \mathrm{pp}}=0.1 \mathrm{~mm}, \mu_{s, \mathrm{pp}}=0.4$, and $d=5 \mathrm{~mm}$. 


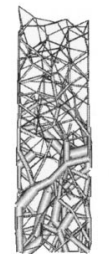

(a)

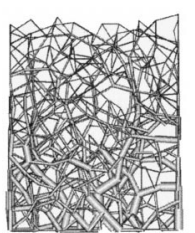

(b)

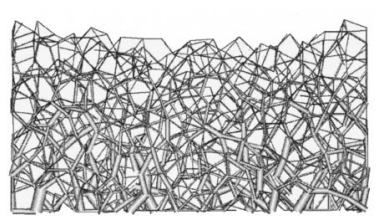

(c)
FIG. 15. Side view of the normal force network ( $5 d$ in depth, cut from center) in sandpiles simulated with $\mu_{r, \mathrm{pp}}=0.1 \mathrm{~mm}, \mu_{s, \mathrm{pp}}$ $=0.4, d=5 \mathrm{~mm}$ and different container thickness $w$ : (a) $w=4 d$; (b) $w=8 d$; (c) $w=16 d$.

the container thickness [Fig. 15(c)]. In that case, the angle of repose is not sensitive to the thickness of container any more (Fig. 13).

It has been reported that the relationship between the angle of repose $\theta$ and and the container thickness $w$ can be described by an exponential law [15]: $\theta=\theta_{0}\left(1+\alpha e^{-\mathrm{kw}}\right)$, where $\alpha$ and $k$ are parameters that depend on particle characteristics and material properties, $\theta_{0}$ is the angle of repose without front and rear wall effect, theoretically obtained when $w=+\infty$. According to this equation, the plot of $(\theta$ $\left.-\theta_{0}\right) / \theta_{0}$ against $w$ should yield a straight line. As shown in Fig. 16, this is indeed the case for the present numerical results. Furthermore, the results suggest that $\alpha=1$ and $k$ $=0.18 / \mathrm{d}$. Therefore, different sized particles give different lines for the plot of $\left(\theta-\theta_{0}\right) / \theta_{0}$ against $w$. This is contrary to the observation of Grasselli and Herrmann [15] that parameter $k$ is independent of the particle size. This result can be due to the different conditions between the experiments and simulations as noted above. However, if particle size is regarded as the only variable responsible for the difference, then the combination of previous experimental and present numerical results suggests that parameter $k$ is constant for small particles and decreases with particle size for large particles as shown in Fig. 17. Further study is necessary to clarify this issue.

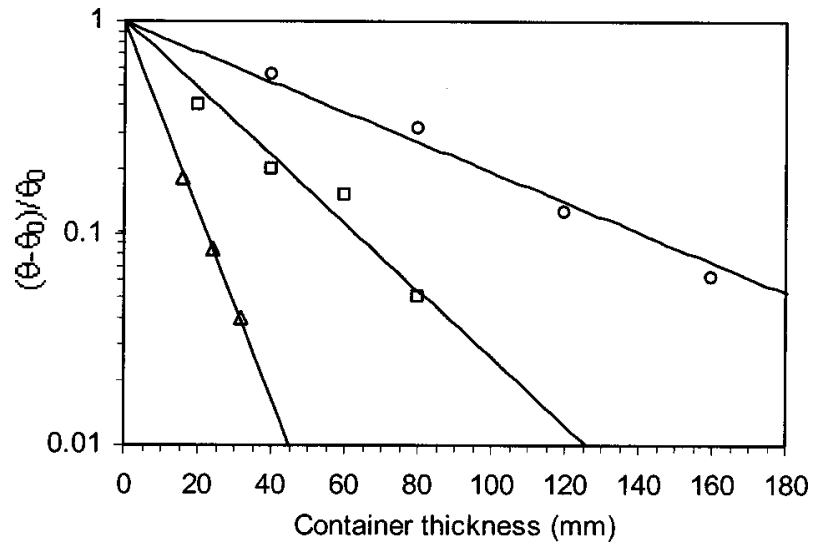

FIG. 16. $\left(\theta-\theta_{0}\right) / \theta_{0}$ vs container thickness $w$ for particles of different sizes simulated when $\mu_{s, \mathrm{pp}}=0.4$ and $\mu_{r, \mathrm{pp}}=0.025 \mathrm{~mm}: \triangle$, $d=2 \mathrm{~mm} ; \square, d=5 \mathrm{~mm} ; \bigcirc, d=10 \mathrm{~mm}$; lines, fitted exponential curves.

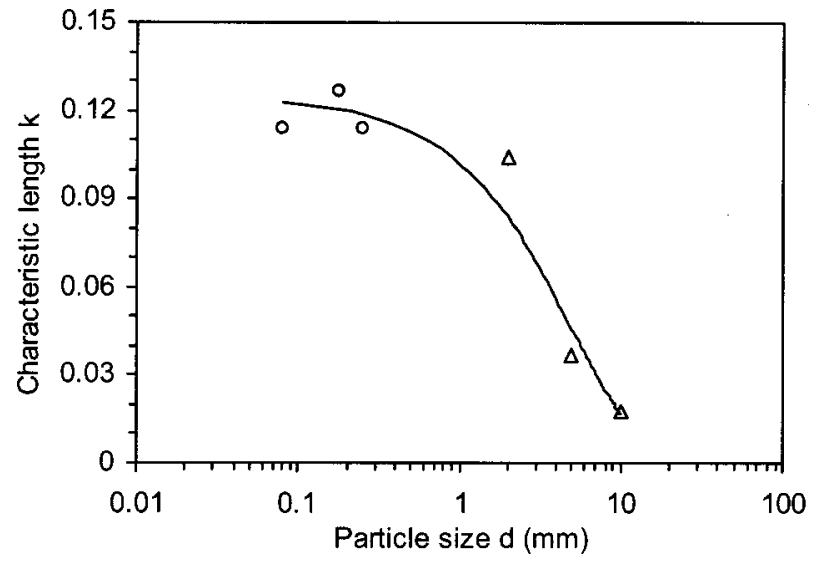

FIG. 17. $k$ vs $d$ : $\bigcirc$, experimental results of Grasselli and Hermann [15]; $\triangle$, present numerical results.

\section{CONCLUSIONS}

A modified DEM simulation technique by incorporating a rolling friction model has been employed to study the formation of sandpiles. The results indicate that the angle of repose is not obviously sensitive to density, Poisson's ratio, damping coefficient and Young's modulus under the present simulation conditions. It is, however, significantly affected by rolling friction coefficient, sliding friction coefficient, particle size, and container thickness, and the effects can be summarized as:

(1) Sliding and rolling frictions among particles and between particle and wall are the primary factors of controlling the translational and rotational motion of a particle and hence the formation of a stable sandpile; the angle of repose increases with the increase of either rolling or sliding friction coefficient.

(2) The angle of repose decreases with the increase of particle size. This effect mainly results from the effect of particle size on rolling friction but not on sliding friction.

(3) Decreasing the container thickness promotes the formation of strong force arches originated from the front and rear walls and hence increases the angle of repose. The relationship between the angle of repose and the container thickness can be described by the exponential law proposed by Grasselli and Herrmann [15]. The characteristic length in this law is not a constant but varies with particle size for coarse particles.

Finally, we would like to point out that based on the present numerical results, empirical equations have been formulated to relate the angle of repose to the above variables for engineering application. Both the simulation technique and the formulated equations have been validated through physical experiments under comparable conditions [28]. This would provide a sound basis for future work more focused on the internal state of sandpiles, e.g., the evolution of the force network in forming a sandpile.

\section{ACKNOWLEDGEMENTS}

The authors are grateful to the Australian Research Council and BHP for financial support of this project. 
[1] J. Lee, J. Phys. I 3, 2017 (1993).

[2] V. Frette, K. Christensen, A. Malthesorenssen, J. Feder, T. Jossang, and P. Meakin, Nature (London) 379, 49 (1996).

[3] H. Jaeger, C. Liu, and S. Negel, Phys. Rev. Lett. 62, 40 (1989).

[4] H. A. Makse, Phys. Rev. A 56, 7008 (1997).

[5] J. Baxter, U. Tuzun, D. Heyes, I. Hayati, and P. Fredlund, Nature (London) 391, 136 (1998).

[6] R. Jullien, P. Meakin, and A. Pavlovitch, Europhys. Lett. 22, 523 (1993).

[7] V. Buchholtz and T. Poschel, Physica A 202, 390 (1994).

[8] C. M. Dury, G. H. Ristow, J. L. Moss, and M. Nakagawa, Phys. Rev. A 57, 4491 (1998).

[9] J. Lee and H. J. Herrmann, J. Phys. A 26, 373 (1993).

[10] K. M. Hill and J. Kakalios, Phys. Rev. A 52, 4393 (1995).

[11] Y. Zhou, B. Wright, R. Yang, B. Xu, and A. Yu, Physica A 269, 536 (1999).

[12] A. Burkalow, Bull. Geol. Soc. Am. 56, 669 (1945).

[13] J. Carstensen and P. Chan, Powder Technol. 15, 129 (1976).

[14] M. Carrigy, Sedimentology 14, 147 (1970).

[15] Y. Grasselli and H. J. Herrmann, Physica A 246, 301 (1997).
[16] H. Kalman, D. Goder, M. Rivken, and G. Ben-Dor, Bulk Solids Handling 13, 123 (1993).

[17] R. Jullien and P. Meakin, Nature (London) 344, 425 (1990).

[18] P. Meakin and R. Jullien, Physica A 180, 1 (1992).

[19] A. Mehta and G. C. Barker, Europhys. Lett. 27, 501 (1994).

[20] A. Mehta and G. C. Barker, Rep. Prog. Phys. 57, 383 (1994).

[21] P. Cundall and O. Strack, Geotechnique 29, 47 (1979).

[22] K. Yamane, M. Nakagawa, S. A. Altobelli, T. Tanaka, and Y. Tsuji, Phys. Fluids 10, 1419 (1998).

[23] T. Elperin and E. Golshtein, Physica A 242, 332 (1997).

[24] J. Baxter, U. Tuzun, J. Burnell, and D. M. Heyes, Phys. Rev. E 55, 3546 (1997).

[25] N. Standish, A. Yu, and Q. He, Powder Technol. 68, 187 (1991).

[26] L. Bocquet, E. Charlaix, S. Ciliberto, and J. Crassous, Nature (London) 396, 735 (1998).

[27] N. Fraysse, H. Thome, and L. Petit, Eur. Phys. J. B 11, 615 (1999).

[28] Y. Zhou, B. Xu, A. Yu and P. Zulli (unpublished). 